\title{
Prevention and Amelioration of Rodent Ventilation-Induced Lung Injury with Either Prophylactic or Therapeutic feG Administration
}

\author{
Alison S. F. Elder ${ }^{1} \cdot$ Andrew D. Bersten $^{1,2} \cdot$ Gino T. P. Saccone $^{3} \cdot$ Claudine S. Bonder $^{4} \cdot$ Dani-Louise Dixon $^{1,2}$ (D)
}

Received: 17 April 2019 / Accepted: 8 July 2019 / Published online: 12 July 2019

(c) Springer Science+Business Media, LLC, part of Springer Nature 2019

\begin{abstract}
Purpose Mechanical ventilation is a well-established therapy for patients with acute respiratory failure. However, up to $35 \%$ of mortality in acute respiratory distress syndrome may be attributed to ventilation-induced lung injury (VILI). We previously demonstrated the efficacy of the synthetic tripeptide feG for preventing and ameliorating acute pancreatitis-associated lung injury. However, as the mechanisms of induction of injury during mechanical ventilation may differ, we aimed to investigate the effect of feG in a rodent model of VILI, with or without secondary challenge, as a preventative treatment when administered before injury (prophylactic), or as a therapeutic treatment administered following initiation of injury (therapeutic). Methods Lung injury was assessed following prophylactic or therapeutic intratracheal feG administration in a rodent model of ventilation-induced lung injury, with or without secondary intratracheal lipopolysaccharide challenge.

Results Prophylactic feG administration resulted in significant improvements in arterial blood oxygenation and respiratory mechanics, and decreased lung oedema, bronchoalveolar lavage protein concentration, histological tissue injury scores, blood vessel activation, bronchoalveolar lavage cell infiltration and lung myeloperoxidase activity in VILI, both with and without lipopolysaccharide. Therapeutic feG administration similarly ameliorated the severity of tissue damage and encouraged the resolution of injury. feG associated decreases in endothelial adhesion molecules may indicate a mechanism for these effects. Conclusions This study supports the potential for feG as a pharmacological agent in the prevention or treatment of lung injury associated with mechanical ventilation.
\end{abstract}

Keywords Acute lung injury $\cdot$ Adhesion molecules $\cdot$ Leukocytes $\cdot$ Mechanical ventilation $\cdot$ Rat

Electronic supplementary material The online version of this article (https://doi.org/10.1007/s00408-019-00252-1) contains supplementary material, which is available to authorized users.

Dani-Louise Dixon

dani.dixon@flinders.edu.au

1 Critical Care Medicine, College of Medicine and Public Health, Flinders University, GPO Box 2100, Adelaide 5001, Australia

2 Intensive and Critical Care Unit, Flinders Medical Centre, Adelaide, Australia

3 Surgery, College of Medicine and Public Health, Flinders University, Adelaide, Australia

4 SA Pathology and the Department of Medicine, Centre for Cancer Biology, University of Adelaide, Adelaide, Australia

\section{Introduction}

Mechanical ventilation is a well-established therapy for patients with acute respiratory failure, however, potential adverse effects include ventilation-induced lung injury (VILI) and barotrauma, ventilator-associated pneumonia, haemodynamic changes, and neuromuscular disorders [1]. As up to $35 \%$ of mortality in acute respiratory distress syndrome (ARDS), may be attributed to VILI, this is an important area of research.

Experimental studies suggest two main mechanisms of VILI: volutrauma, injury from excess distension; and atelectrauma, local shear injury from tidal opening and closing. In the rat model of VILI, high tidal volume and zero positive end-expiratory pressure (PEEP) induce physiological and morphological lung changes, with altered cellular processes within the lung [2]. Hyperdistension of lung parenchyma is transmitted from the extracellular environment to the cell interior through the extracellular matrix-cytoskeleton [1], 
resulting in plasma membrane and barrier dysfunction, a primary factor in VILI [3]. VILI also exacerbates respiratory dysfunction due to pulmonary alveolar inflammation resulting from lipopolysaccharide (LPS) exposure alone [4, 5]. Previously, we have utilized models of VILI with secondary challenge to demonstrate the presentation of lung injury immediately following commencement of VILI [6].

The ability of a novel therapeutic pharmacological agent, the synthetic tripeptide feG ((D-Phe)-(D-Glu)-Gly), to decrease neutrophil recruitment, infiltration, and activation in vitro and in vivo has previously been demonstrated [7]. Possible mechanisms include decrease in integrin and adhesion molecule expression, and direct anti-inflammatory responses including decrease in reactive oxygen species and inflammatory cytokine production on neutrophils [7-9]. Dose-response assays have demonstrated a bell-shaped response curve, with neither lower $(10 \mu \mathrm{g} / \mathrm{kg})$ or higher $(350 \mu \mathrm{g} / \mathrm{kg})$ peptide doses inhibiting neutrophil migration in various models of injury [7]. Utilizing models of acute pancreatitis-associated lung injury we have demonstrated both prevention and amelioration of lung injury following prophylactic or therapeutic feG administration $[8,10,11]$. However, the ability of feG to similarly prevent or ameliorate mechanically induced lung damage such as in VILI has not been previously investigated.

We hypothesised that feG would reduce the recruitment, infiltration, and/or activation of neutrophils, during VILI, with or without secondary LPS challenge. In addition, we hypothesised that this reduction in neutrophil infiltration into the lung may be due to inhibition of endothelial cell surface adhesion molecules. Thus we investigated the effect of feG when administered before (prophylactic) or after (therapeutic) induction of VILI, with and without secondary LPS challenge, on the severity of tissue damage and resolution of injury.

\section{Materials and Methods}

Please refer to the online supplement for greater detail.

\section{Animals}

Male Sprague-Dawley rats (100-130 g) were randomly allocated to treatment groups (Table S1; $n=4-7$ ). The study protocol was approved by the Flinders University Animal Welfare Committee.

Rats were anaesthetised via continuous intravenous infusion of thiopental $(60 \mathrm{mg} / \mathrm{kg} / \mathrm{h}$; Abbott) and paralysed with a bolus injection of pancuronium bromide ( $1 \mathrm{mg} / \mathrm{kg}$ i.v.; Astra Zeneca, UK) maintained by continuous infusion $(0.2 \mathrm{mg} /$ $\mathrm{kg} / \mathrm{h}$ i.v.). Mean arterial pressure was continuously monitored through cannulation of the left femoral artery using disposable blood pressure transducers connected to a PowerLab and Quad Bridge Amp (AD Instruments, Australia).

\section{VILI}

VILI was induced by $2 \mathrm{~h}$ of injurious ventilation (high tidal volume $(\mathrm{Vt})=35 \mathrm{ml} / \mathrm{kg}$; positive end-expiratory pressure $(\mathrm{PEEP})=0 \mathrm{cmH}_{2} \mathrm{O}$; fraction of inspired oxygen $\left(\mathrm{FiO}_{2}\right)=$ 1.0; breathing frequency $\left.(f)=20 \mathrm{~min}^{-1}\right)$. The control group received low-Vt non-injurious ventilation $(\mathrm{Vt}=7 \mathrm{ml} / \mathrm{kg}$; $\mathrm{PEEP}=2 \mathrm{cmH}_{2} \mathrm{O} ; \mathrm{FiO}_{2}=1.0 ; f=120 \mathrm{~min}^{-1}$ ).

\section{VILI + LPS}

A tracheotomy was performed before intratracheal instillation of lipopolysaccharide (LPS) (Escherichia coli O55:B5, $2 \mathrm{mg} / \mathrm{kg}$ body weight in saline; Sigma-Aldrich, MO) [6, 11]. VILI was then induced by $2 \mathrm{~h}$ of injurious ventilation, as previously. Control rats received equivalent volumes of saline intratracheally (vehicle control) and received low-Vt non-injurious ventilation, as previously.

\section{feG Administration}

To investigate the effect of feG as a preventative and therapeutic treatment, on the severity of tissue damage and resolution of injury, when administered (1) before (prophylactic) or (2) after VILI (therapeutic), (3) with or (4) without secondary LPS challenge,

A single $0.1 \mathrm{ml}$ dose of feG $(100 \mu \mathrm{g} / \mathrm{kg}$ body weight in saline; kindly supplied by Dr Joseph Davison, $\mathrm{PhD}$, University of Calgary, Canada) was administered intratracheally 5 min before VILI (prophylactically, feG(0)), or 20 min after initiation of VILI (therapeutically, feG(20)). Vehicle control groups received intratracheal saline.

\section{Respiratory Mechanics}

Respiratory mechanics were assessed after $2 \mathrm{~h}$ by measuring the lung's impedance following a forced oscillation using a computer-controlled ventilator [12].

\section{Lung Harvest}

Following $2 \mathrm{~h}$ of ventilation, the lungs and the heart was removed en bloc. Lung lobes were resected for determination of wet-to-dry weight ratio, MPO activity, and tissue protein determined by colorimetric assay (Bio-Rad Laboratories, $\mathrm{CA}$ ) [13]. Bronchoalveolar lavage (BAL) was performed, following isolation of the top right two lobes, for determination of alveolar cytokine, total protein and cells [13]. 


\section{Histological and Immunohistological Analysis}

Following BAL, the right lung lobes were fixed at $20 \mathrm{cmH}_{2} \mathrm{O}$ with $10 \%$ buffered formalin, paraffin-embedded and cut into $4 \mu \mathrm{m}$ sections. Sections were stained with hematoxylin and eosin [6] for scoring of pulmonary inflammatory cell infiltration and alveolar wall thickening using a semiquantitative score (0-3) on blinded, randomly selected images by two independent investigators [14].

Similarly, formalin-fixed, paraffin-embedded sections were labelled with vascular cell adhesion molecule (VCAM)-1 antibody (C-19, Santa Cruz (sc-1504), $10 \mu \mathrm{g} / \mathrm{ml}$ ) or PECAM-1 (M-20, Santa Cruz (sc-1506), $0.2 \mu \mathrm{g} / \mathrm{ml}$ ) and counterstained with hematoxylin before visualisation by an Olympus BX45 microscope linked to a XC10 camera. Five images were collected per sample and were assessed using a semiquantitative score as above for intensity $(0-3)$ and density of staining ( $0-3)$, on blinded sections by two independent investigators. To ensure specificity of the immunohistological staining reactions, consecutive sections were incubated in the absence of the primary antibody.

\section{Analysis}

The main outcome measures were arterial blood oxygenation, respiratory compliance, lung oedema, lung tissue protein concentration, BAL soluble protein concentration, histological tissue injury scores, BAL cell infiltration and lung MPO activity. Due to small group sizes, difference between groups was tested by nonparametric Kruskal-Wallis test followed by Mann-Whitney U post hoc analyses, as appropriate, using SPSS v17.0 (IBM, NY) and data are displayed as column graphs (mean $\pm \mathrm{SD}$ ). A $p \leq 0.05$ was considered statistically significant.

\section{Results}

\section{VILI}

High-Vt mechanical ventilation decreased arterial blood oxygenation, and increased respiratory tissue elastance, lung oedema, lung tissue protein concentration, histological tissue injury scores, BAL cell infiltration and lung MPO activity (Figs. 1 and 2, and S1) (Table 1). The VILI alone model did not result in a change in BAL soluble protein concentration, however, a small increase was found in soluble protein from homogenised lung tissue following induction of VILI, compared to baseline control values (Fig. 1d).

Prophylactic feG treatment (High Vt $+\mathrm{feG}(0)$ ) significantly improved arterial blood oxygenation and respiratory mechanics, and decreased lung oedema, lung tissue protein concentration, histological tissue injury scores, BAL cell infiltration and lung MPO activity, most to baseline control values (Figs. 1 and 2, and S1) (Table 2). Therapeutic feG treatment (High Vt $+\mathrm{feG}(20)$ ) similarly improved these indices of lung injury, most to baseline saline control values.

Investigation of vascular adhesion molecules in the feG treatment groups (High $\mathrm{Vt}+\mathrm{feG}(0)$ and $\mathrm{High} \mathrm{Vt}+\mathrm{feG}(20)$ ) identified a significant decrease in vascular cell adhesion molecule (VCAM)-1 and platelet endothelial cell adhesion molecule (PECAM)-1 in the animals treated with feG either prophylactically or therapeutically (Fig. 3) (Table 2). No immunostaining was detected in the antibody control.

\section{VILI + LPS}

The addition of LPS exacerbated lung injury with decreased arterial blood oxygenation, diminished respiratory compliance, and increased BAL protein concentration and histological tissue injury scores, compared with VILI alone (Fig. S2). High-Vt mechanical ventilation with intratracheal LPS instillation decreased arterial blood oxygenation, diminished respiratory compliance, and increased lung oedema, BAL protein concentration, histological tissue injury scores, BAL cell infiltration and lung MPO activity (Figs. 2 and 4, and S3) (Table 2). Unlike the VILI alone model, the combination of high-Vt mechanical ventilation with intratracheal LPS resulted in increased BAL soluble protein concentration, due to the exacerbation of VILI and generation of lung inflammation (Fig. 4d).

Prophylactic feG treatment (LPS + High Vt $+\mathrm{feG}(0)$ ) significantly improved arterial blood oxygenation and respiratory mechanics, and decreased lung oedema, histological tissue injury scores, BAL neutrophil and macrophage infiltration and lung MPO activity, most to baseline control values (Figs. 2 and 4, and S3) (Table 2). Therapeutic feG treatment (LPS + High Vt+feG(20)) similarly improved respiratory mechanics, and decreased BAL protein concentration, histological tissue injury scores, and BAL neutrophil and macrophage infiltration.

\section{Discussion}

In this study, feG reduced leukocyte infiltration, ameliorated the severity of lung injury, and restored lung function when administered both prophylactically and therapeutically. In addition, a 'two-hit' presentation of secondary bacterial infection on a background of VILI, modelled in this study through the intratracheal instillation of LPS, similarly resulted in amelioration and resolution of lung injury, following prophylactic or therapeutic feG administration. We previously established the efficacy of feG in inhibiting the inflammatory response and ameliorating and preventing injury in classically inflammatory models of acute lung 
injury $[8,11]$. Amelioration of lung injury in VILI, which is primarily a mechanically induced lung injury model, albeit with recognised inflammatory components [3], provides valuable additional insights into the mechanisms of action of feG.

VILI can induce physiological and morphological lung changes, with altered cellular processes within the lung [2]. Previously, we have demonstrated lung injury that is apparent immediately upon commencement of VILI, with diminished respiratory function and decreased arterial blood oxygenation. Mechanisms contributing to VILI include
Fig. 2 Histological improvement of lung damage following prophylactic or therapeutic feG treatment. Images of hematoxylin and eosin-stained sections of lung tissue harvested from rats following prophylactic (0) or therapeutic (20) feG administration in the a ventilation-induced lung injury (VILI) model, and b lipopolysaccharide (LPS) + VILI model. Magnification $\times 40$

regional overdistension, resulting from the application of stress that causes tissues and cells to assume configurations not found in unaided breathing [15], and low-volume injury, which results from the repeated collapse and recruitment of
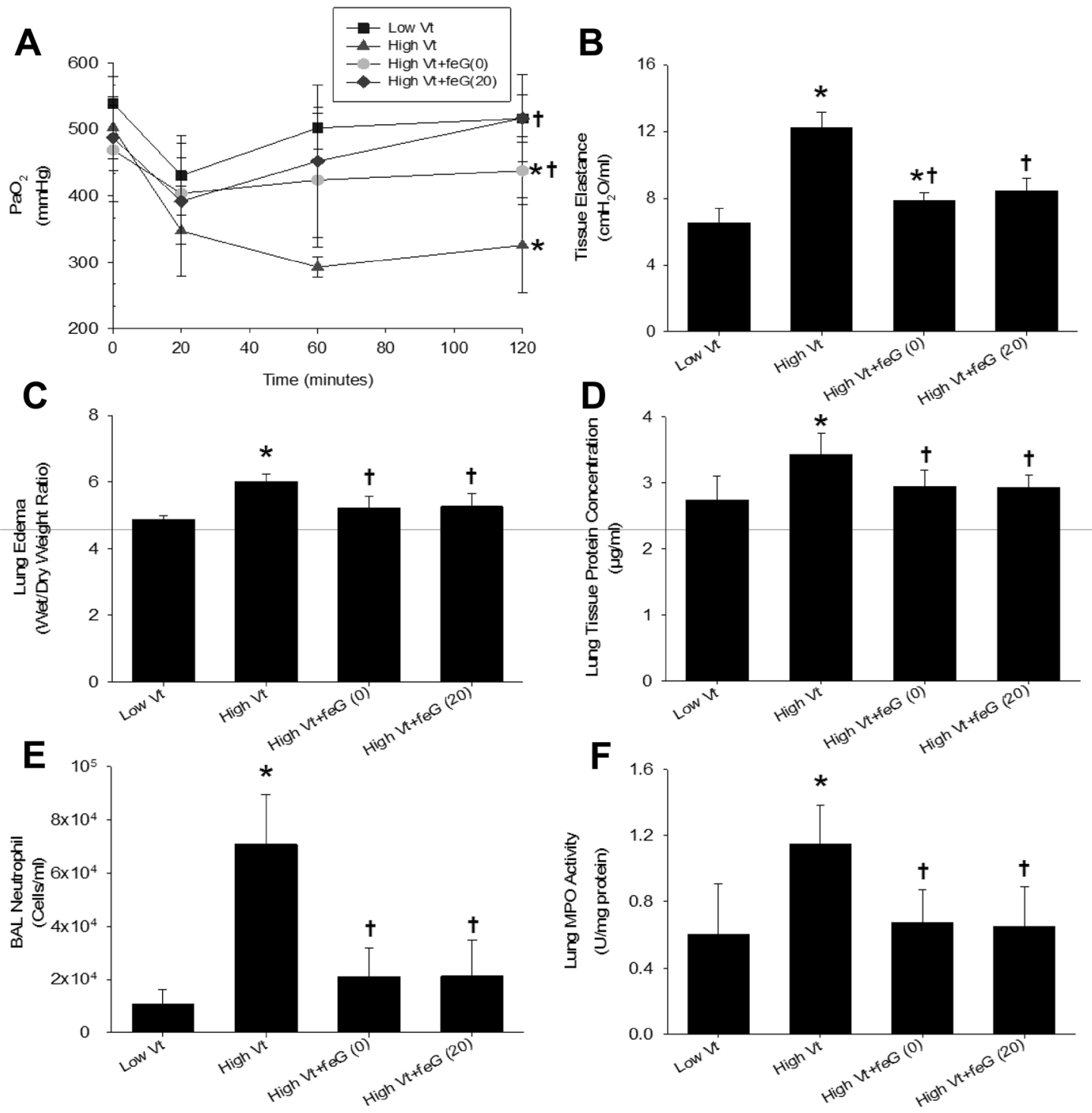

Fig. 1 Ventilation-induced lung injury (VILI) induces severe lung damage which is ameliorated following feG treatment. Changes in arterial blood $\mathrm{PaO}_{2}(\mathrm{mmHg})$, tissue elastance $\left(\mathrm{cmH}_{2} \mathrm{O} / \mathrm{ml}\right)$, lung oedema (wet/dry weight ratio), lung tissue protein concentration $(\mu \mathrm{g} /$ $\mathrm{ml})$, bronchoalveolar lavage (BAL) neutrophil count (cells/ml), and

lung myeloperoxidase (MPO) activity (U/mg protein) following VILI with prophylactic (0) or therapeutic (20) feG administration. Data are presented as mean $\pm \mathrm{SD}(n=4-6)$. ${ }^{*} p<0.05$ compared with low $\mathrm{Vt}$. $\dagger p<0.05$ compared with High Vt 

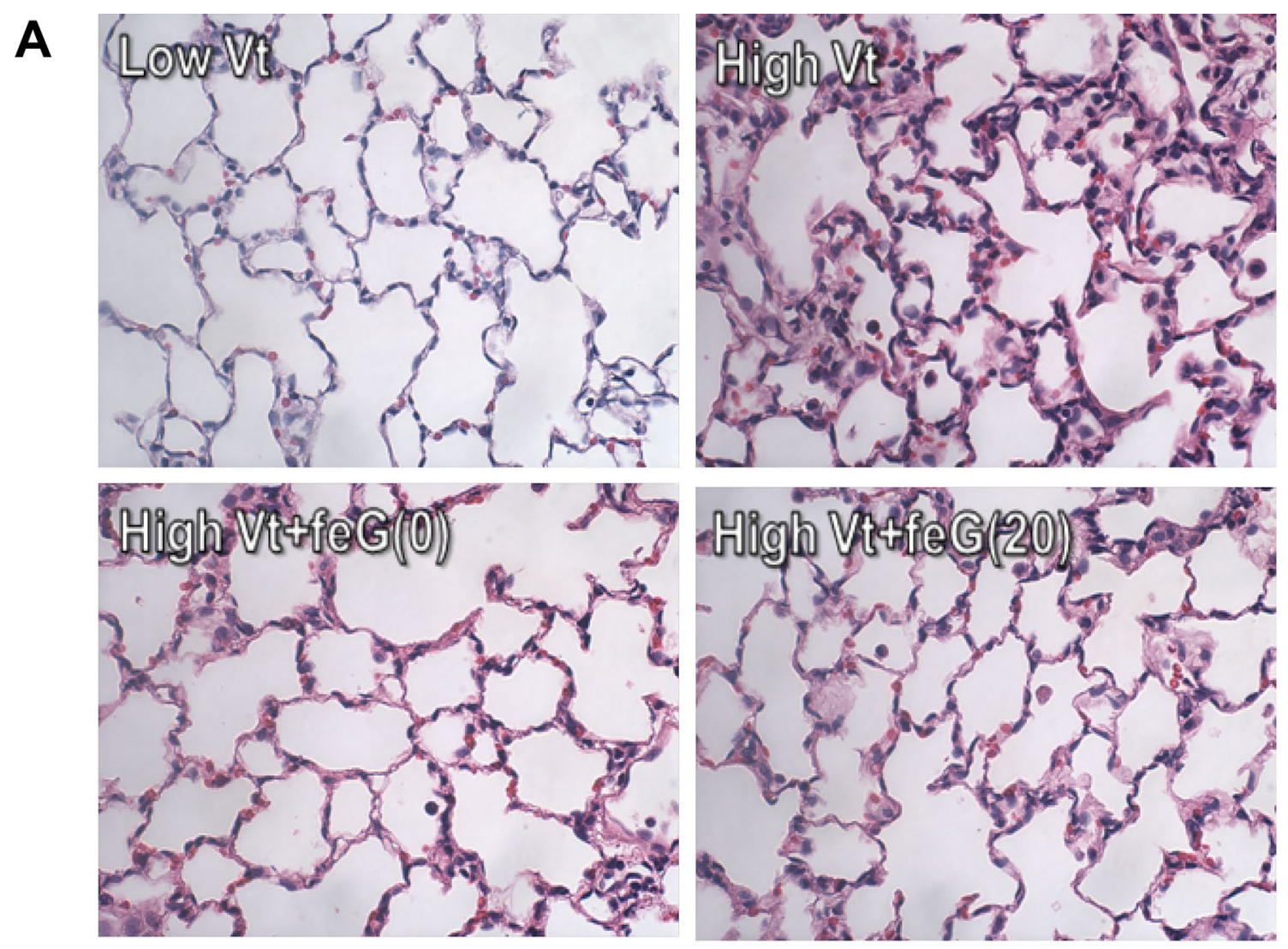

\section{B}
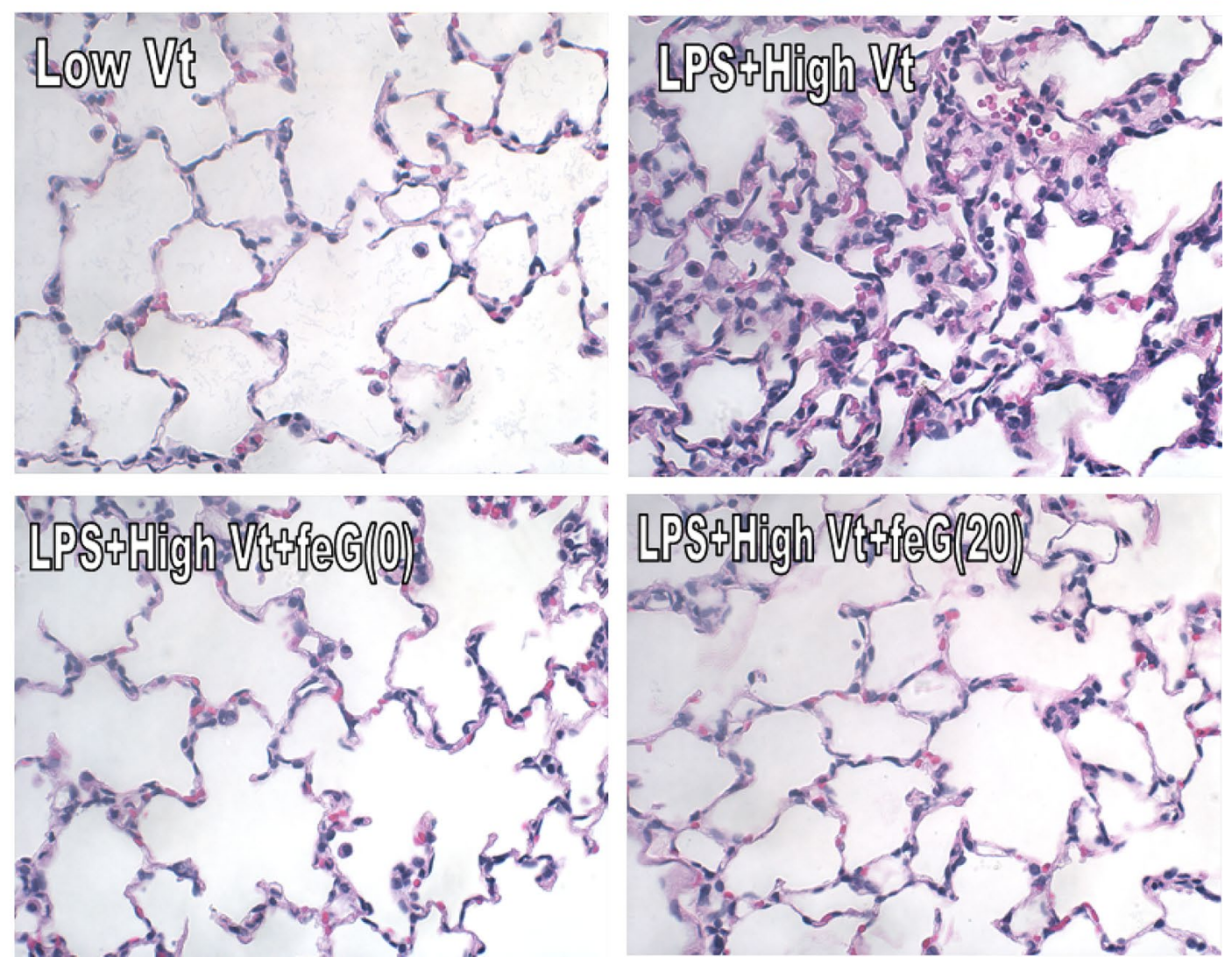
Table 1 Histological lung alteration scores following ventilation-induced lung injury (VILI), with or without intratracheal lipopolysaccharide (LPS) instillation, and prophylactic or therapeutic feG administration

\begin{tabular}{|c|c|c|c|c|}
\hline Model & Group & $n$ & $\begin{array}{l}\text { Pulmonary inflamma- } \\
\text { tory cell infiltration }\end{array}$ & Alveolar wall thickening \\
\hline \multirow[t]{4}{*}{ VILI } & Low Vt & 4 & $0.92 \pm 0.69$ & $0.83 \pm 0.43$ \\
\hline & High Vt & 5 & $2.13 \pm 0.51 *$ & $2.20 \pm 0.38^{*}$ \\
\hline & High Vt + feG $(0)$ & 6 & $1.44 \pm 0.54$ & $1.39 \pm 0.25 \#$ \\
\hline & High Vt + feG (20) & 6 & $1.72 \pm 0.53$ & $1.56 \pm 0.17 * \#$ \\
\hline \multirow[t]{4}{*}{ VILI + LPS } & Low Vt & 6 & $0.89 \pm 0.27$ & $0.84 \pm 0.55$ \\
\hline & LPS + High Vt & 7 & $2.67 \pm 0.39 *$ & $2.33 \pm 0.27 *$ \\
\hline & LPS + High Vt + feG (0) & 6 & $1.17 \pm 0.28 \#$ & $1.45 \pm 0.35 * \#$ \\
\hline & LPS + High Vt + feG (20) & 7 & $1.50 \pm 0.28 * \#$ & $1.50 \pm 0.28^{* \#}$ \\
\hline
\end{tabular}

Table 2 Vascular cell adhesion molecule (VCAM)-1 and platelet endothelial cell adhesion molecule (PECAM)-1 intensity and density of staining scores following ventilation-induced lung injury with prophylactic or therapeutic feG administration

\begin{tabular}{llllll}
\hline $\begin{array}{l}\text { Vascular adhesion } \\
\text { molecules }\end{array}$ & \multicolumn{2}{l}{ Groups } & & & \\
\cline { 2 - 6 } & Low Vt & High Vt & High Vt + feG $(0)$ & High Vt + feG $(20)$ & $p$ \\
\hline VCAM-1 & $3.3 \pm 1.5$ & $6.0 \pm 0.0^{*}$ & $3.0 \pm 1.6 \#$ & $2.8 \pm 1.9 \#$ & 0.05 \\
PECAM-1 & $2.3 \pm 1.0$ & $4.0 \pm 1.0^{*}$ & $2.3 \pm 1.0 \#$ & $1.4 \pm 0.5 \#$ & 0.01 \\
\hline
\end{tabular}

Data are presented as mean \pm SD $(n=3-6)$

${ }^{*} p<0.05$ compared with Low Vt. \#p $<0.05$ compared with High Vt

unstable lung structure leading to epithelial airspace lining abrasion (atelectrauma) [15]. Resulting mechanotransduction, a chemical signal production by cells in response to mechanical stimuli, leads to alveolar oedema and accumulation of plasma proteins, surfactant dysfunction and increased surface tension resulting in increased tissue resistance and tissue elastance and subsequent impairment in gas exchange [3]. In ALI, mechanical ventilation can exacerbate pre-existing lung injury, as well as initiating lung injury, through the interaction of reparative and injurious mechanotransductive pathways. VILI thereby can result in disruption of pulmonary epithelium and endothelium, atelectasis, hypoxemia, and release of inflammatory mediators [16]. The VILI model, therefore, contrasts with ALI models induced by endotoxin or secondary to inflammatory diseases, including acute pancreatitis, whereby the initiating and predominant insult is inflammatory with chemotactic cytokine-mediated infiltration of large numbers of activated neutrophils to the lung $[8,11]$. Together with resident macrophages, these neutrophils facilitate an inflammatory cascade, stimulating both further leukocyte chemotaxis and a respiratory burst, resulting in pulmonary epithelial cell apoptosis and necrosis $[11,17]$. While the ultimate end-points of pulmonary epithelial barrier permeability, influx of protein-rich oedematous fluid and surfactant inactivation, resulting in decreased lung compliance and impaired gas exchange [17-19], remain similar, there is an intrinsic difference, primarily in neutrophil response [8]. Additionally, increased soluble protein in homogenised lung tissue, but not BAL, was noted in this study. This increase in soluble tissue protein may be due to deposition within the interstitial spaces with a smaller intra-alveolar flux. Our observed amelioration in the severity of lung injury, and resolution of lung injury, following feG administration in both VILI and VILI with severe secondary lung challenge resulting in pronounced lung inflammation, indicate that in the clinical setting feG may be administered either as a preventative at the commencement of mechanical ventilation, or as a treatment following the development of ARDS.

Maintaining adequate peripheral oxygenation without increasing tidal volume is one of the main challenges in the intensive care unit, and the primary criteria for lung-protective ventilation strategies [20]. Despite significant advances in injury prevention with these modified ventilation strategies [16], which include reduced tidal volume and addition of PEEP, morbidity and mortality due to VILI-associated acute lung injury remain, highlighting the complexity of this condition [3,21]. Mortality rates associated with VILI have been reduced by $22 \%$ via the reduction of tidal volume from $12 \mathrm{ml} / \mathrm{kg}$ to $6 \mathrm{ml} / \mathrm{kg}$ in critically ill patients [16]. The addition of PEEP is a crucial component of non-injurious mechanical ventilation [22], as it improves oxygenation by recruiting collapsed alveoli and increasing the functional residual capacity. However, these strategies alone do not provide complete protection against VILI [23]. A recent study observed substantial over distension, via computed tomography, and inflammatory mediator release in one-third of ARDS patients ventilated with a tidal volume of $6 \mathrm{ml} /$ 
Fig. 3 Significantly decreased vascular cell adhesion molecule (VCAM)-1 and platelet endothelial cell adhesion molecule (PECAM)-1 following prophylactic or therapeutic feG treatment. Images of lung tissue harvested from rats in the ventilation-induced lung injury model. Magnification $\times 40$

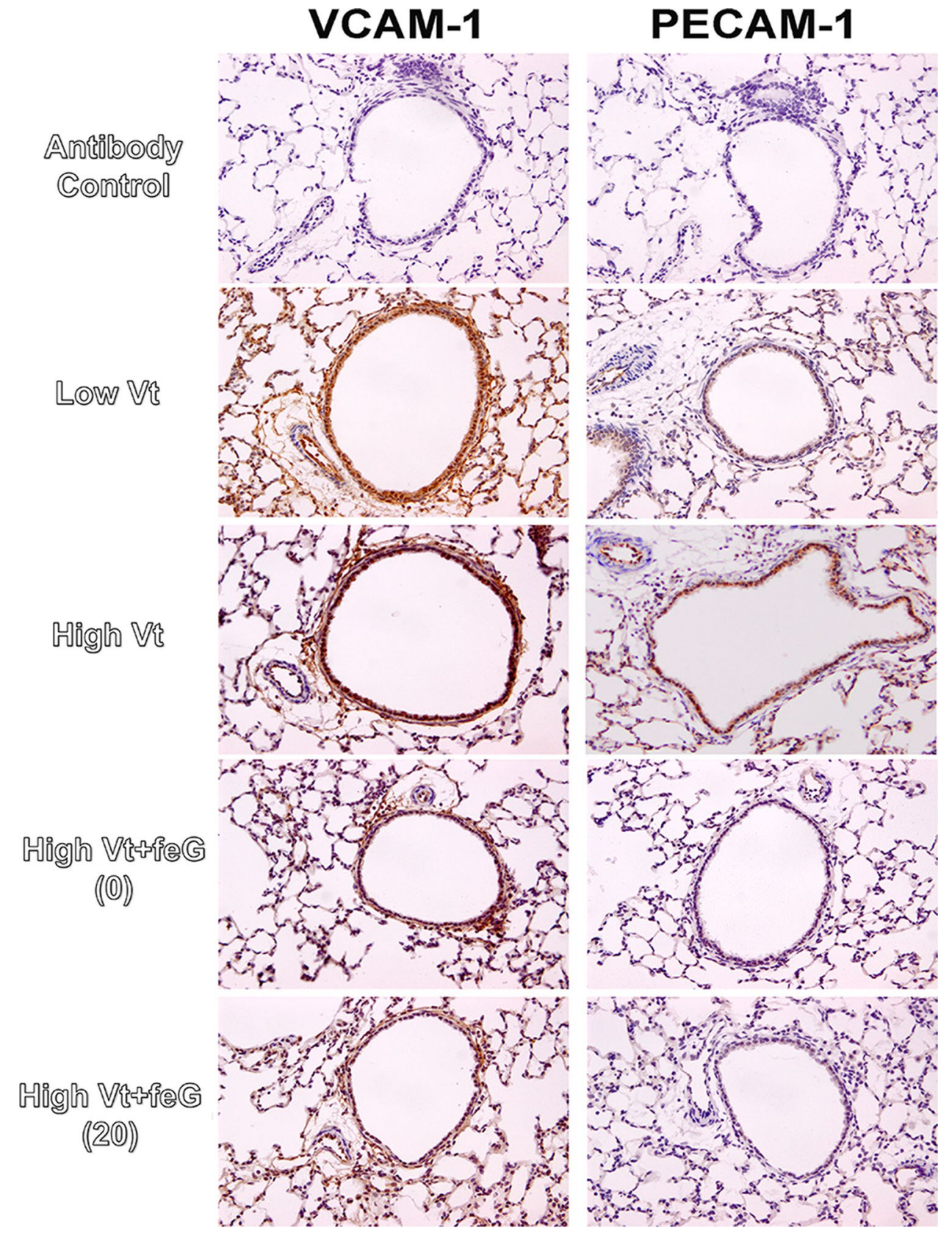

kg predicted body weight [24]. Stress distribution within the lung is also not uniform and therefore identical ventilation parameters in two individual patients can result in variable amounts of mechanotransduction [3]. Therapies are, therefore, still needed to alleviate lung injury and multiple systemic organ failure secondary to VILI. feG appears promising as a preventative treatment at the commencement of mechanical ventilation, following the development of VILI, or as a preventative against additional injury due to secondary infection. In addition, the administration of feG prophylactically to vulnerable ventilated patients would mean that tidal volume could be safely increased if augmented alveolar ventilation were indicated.
The synthetic analogue, feG, is derived from the submandibular gland peptide-T, which is a biologically active sevenamino acid peptide that protects against systemic inflammatory response syndrome [7]. Previous dose-response assays have demonstrated a bell-shaped dose-response curve, with neither lower $(10 \mu \mathrm{g} / \mathrm{kg})$ or higher $(350 \mu \mathrm{g} / \mathrm{kg})$ peptide doses inhibiting neutrophil migration in various models of injury, including endotoxin and ovalbumin challenge [7]. A bell-shaped dose-dependent recovery was also observed in the ability of migrated neutrophils to generate superoxide anion [7]. Previous mechanistic studies using data mining, molecular modelling, and enzymatic techniques have demonstrated that in vitro feG inhibits expression of CD49d on 


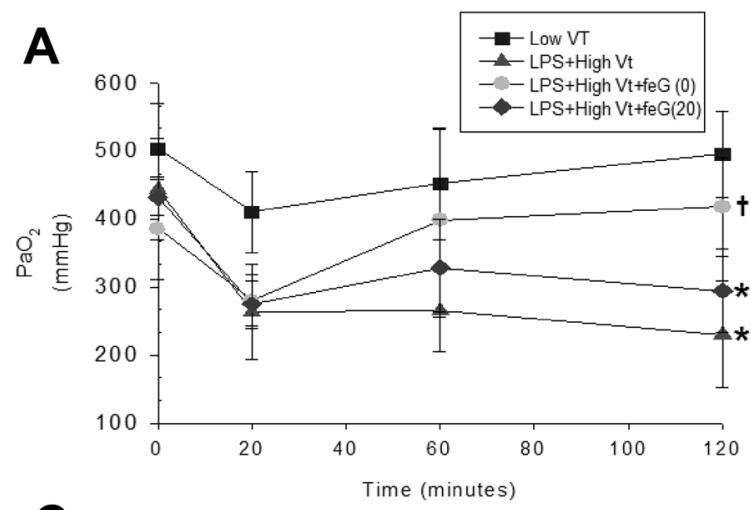

B

C
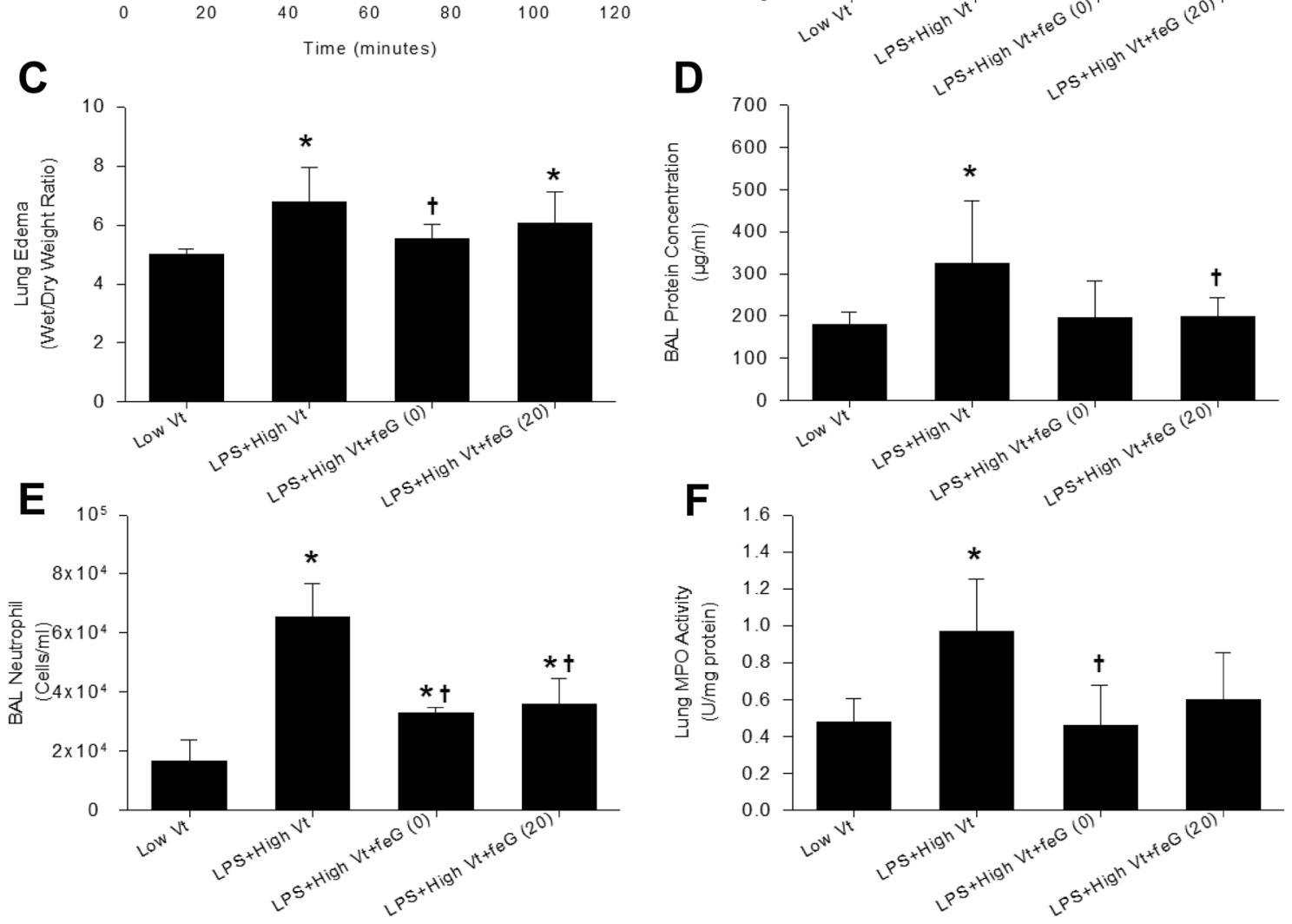

Fig. 4 Ventilation-induced lung injury (VILI) with intratracheal lipopolysaccharide (LPS) induces severe lung inflammation and damage which is ameliorated following prophylactic or therapeutic feG treatment. Changes in arterial blood $\mathrm{PaO}_{2}(\mathrm{mmHg})$, tissue elastance $\left(\mathrm{cmH}_{2} \mathrm{O} / \mathrm{ml}\right)$, lung oedema (wet/dry weight ratio), lung tissue protein concentration $(\mu \mathrm{g} / \mathrm{ml})$, bronchoalveolar lavage (BAL) neutrophil

rat-circulating neutrophils and the binding of $\mathrm{CD} 11 \mathrm{~b}$ and $\mathrm{CD} 16 \mathrm{~b}$ antibodies to stimulated human and rat neutrophils [7, 9]. In vivo feG also reduces the capacity of neutrophils to generate reactive oxygen species, by preventing the deregulation of protein kinase $C[7,9]$. Targeted rat inflammatory gene arrays from a 'two-hit' model of acute pancreatitisinduced lung injury have likewise demonstrated that feG acts by downregulating several inflammatory genes (via direct or indirect methods) that have integrated roles in inflammatory cell trafficking via adhesion, infiltration and activation [8]. From the current study, in line with previous data,

count (cells/ml), and lung myeloperoxidase (MPO) activity (U/mg protein) following VILI with LPS instillation and prophylactic (0) or therapeutic (20) feG administration. Data are presented as mean \pm SD $(n=6-7) .{ }^{*} p<0.05$ compared with low Vt. $\dagger p<0.05$ compared with LPS + High Vt

feG administration is associated with a modulation of the immune response with decreased adhesion molecule expression and neutrophil infiltration into the lungs. Specifically, we investigated vascular adhesion molecule expression and demonstrated that feG downregulates VCAM-1 and PECAM-1 in the lung. Vascular cell adhesion molecule (VCAM)-1, whose expression on pulmonary vascular endothelium is responsible for neutrophil sequestration, recruiting and directing leukocyte traffic to sites of inflammation, was decreased following prophylactic or therapeutic feG administration [25]. Similarly, PECAM-1, whose 
expression on endothelial cell intercellular junctions in confluent vascular beds is responsible for leukocyte recruitment and adhesion, was decreased following prophylactic or therapeutic feG administration [26]. While the previous data indicates that this decrease in vascular adhesion molecule expression may be only part of the overall action of feG, in mice models of acute pancreatitis, blocking VCAM-1 on pulmonary vascular endothelium similarly decreased leukocyte adherence and recruitment into the lung and reduced lung injury, demonstrating the potential importance of this mechanism [25].

In conclusion, these data highlight the potential of feG to act as a preventative treatment when administered before VILI, or as a therapeutic treatment to ameliorate the severity of tissue damage and improve outcomes when administered following initiation of VILI, with or without secondary inflammatory challenge. Therefore, this study indicates that feG may be able to significantly reduce the morbidity and mortality rate associated with VILI. This study supports the potential for feG as a pharmacological agent in the prevention and alleviation of lung injury and multiple systemic organ failure secondary to mechanical ventilation.

Acknowledgements The study was funded by the Flinders Medical Centre Foundation. We thank Samantha Escarbe for technical assistance in immunohistochemical staining of lung sections.

Funding This study was funded by the Flinders medical Centre Foundation.

\section{Compliance with Ethical Standards}

Conflict of interest The authors declare that they have no conflict of interest.

Ethical Approval All applicable international, national, and/or institutional guidelines for the care and use of animals were followed. All procedures performed in studies involving animals were in accordance with the ethical standards of the institution at which the studies were conducted.

\section{References}

1. Taniguchi LU, Caldini EG, Velasco IT, Negri EM (2010) Cytoskeleton and mechanotransduction in the pathophysiology of ventilator-induced lung injury. J Bras Pneumol 36:363-371

2. Kuebler WM (2010) From a distance: ventilation-dependent extrapulmonary injury. Transl Res 155:217-219

3. Plataki M, Hubmayr RD (2010) The physical basis of ventilatorinduced lung injury. Expert Rev Resp Med 4:373-385

4. van Westerloo DJ, Schultz MJ, Bruno MJ, de Vos AF, Florquin S, van der Poll T (2004) Acute pancreatitis in mice impairs bacterial clearance from the lungs, whereas concurrent pneumonia prolongs the course of pancreatitis. Crit Care Med 32:1997-2001

5. Kurahashi K, Kajikawa O, Sawa T, Ohara M, Gropper MA, Frank DW, Martin TR, Wiener-Kronish JP (1999) Pathogenesis of septic shock in Pseudomonas aeruginosa pneumonia. J Clin Investig 104:743-750

6. Dixon DL, De Smet HR, Bersten AD (2009) Lung mechanics are both dose and tidal volume dependant in LPS-induced lung injury. Respir Physiol Neurobiol 167:333-340

7. Mathison RD, Davison JS, Befus AD, Gingerich DA (2010) Salivary gland derived peptides as a new class of anti-inflammatory agents: review of preclinical pharmacology of C-terminal peptides of SMR1 protein. J Inflamm 7:49

8. Elder AS, Bersten AD, Saccone GT, Dixon D-L (2013) Tripeptide feG prevents and ameliorates acute pancreatitis-associated acute lung injury in a rodent model. CHEST J 143:371-378

9. Mathison RD, Christie E, Davison JS (2008) The tripeptide feG inhibits leukocyte adhesion. J Inflamm 5:6

10. Rifai Y, Elder ASF, Carati CJ, Hussey DJ, Li X, Woods CM, Schloithe AC, Thomas AC, Mathison RD, Davison JS, Toouli J, Saccone GTP (2008) The tripeptide analog feG ameliorates severity of acute pancreatitis in a caerulein mouse model. Am J Physiol Gastrointest Liver Physiol 294:G1094-G1099

11. Elder AS, Bersten AD, Saccone GT, Dixon DL (2013) Prevention and amelioration of rodent endotoxin-induced lung injury with administration of a novel therapeutic tripeptide feG. Pulm Pharmacol Ther 26:167-171

12. Davidson KG, Bersten AD, Barr HA, Dowling KD, Nicholas TE, Doyle IR (2002) Endotoxin induces respiratory failure and increases surfactant turnover and respiration independent of alveolocapillary injury in rats. Am J Respir Crit Care Med 165:1516-1525

13. Elder AS, Saccone GT, Bersten AD, Dixon DL (2011) L-Arginine-induced acute pancreatitis results in mild lung inflammation without altered respiratory mechanics. Exp Lung Res 37:1-9

14. dos Santos CC, Shan Y, Akram A, Slutsky AS, Haitsma JJ (2011) Neuroimmune regulation of ventilator-induced lung injury. Am J Respir Crit Care Med 183:471-482

15. Hubmayr RD (2005) Ventilator-induced lung injury without biotrauma? J Appl Physiol 99:384-385

16. Network The Acute Respiratory Distress Syndrome (2000) Ventilation with lower tidal volumes as compared with traditional tidal volumes for acute lung injury and the acute respiratory distress syndrome. New Engl J Med 342:1-8

17. Bhatia M, Moochhala S (2004) Role of inflammatory mediators in the pathophysiology of acute respiratory distress syndrome. $\mathrm{J}$ Pathol 202:145-156

18. Cortjens B, De Boer OJ, De Jong R, Antonis AFG, Sabogal Piñeros YS, Lutter R, Van Woensel JBM, Bem RA (2016) Neutrophil extracellular traps cause airway obstruction during respiratory syncytial virus disease. J Pathol 238:401-411

19. Muhs BE, Patel S, Yee H, Marcus S, Shamamian P (2001) Increased matrix metalloproteinase expression and activation following experimental acute pancreatitis. J Surg Res 101:21-28

20. Diaz JV, Brower R, Calfee CS, Matthay MA (2010) Therapeutic strategies for severe acute lung injury. Crit Care Med 38:1644-1650

21. Barisione C, Garibaldi S, Ghigliotti G, Fabbi P, Altieri P, Casale MC, Spallarossa P, Bertero G, Balbi M, Corsiglia L, Brunelli C (2010) CD14CD16 monocyte subset levels in heart failure patients. Dis Mark 28:115-124

22. Hasan A (2010) Understanding mechanical ventilation: a practical handbook 2nd, edn edn. Springer, New York

23. Del Sorbo L, Slutsky AS (2011) Acute respiratory distress syndrome and multiple organ failure. Curr Opin Crit Care 17:1-6

24. Terragni PP, Rosboch G, Tealdi A, Corno E, Menaldo E, Davini O, Gandini G, Herrmann P, Mascia L, Quintel M, Slutsky AS, Gattinoni L, Ranieri VM (2007) Tidal hyperinflation during low 
tidal volume ventilation in acute respiratory distress syndrome. $\mathrm{J}$ Respir Crit Care Med 175:160-166

25. Callicutt CS, Sabek O, Fukatsu K, Lundberg AH, Gaber L, Wilcox H, Kotb M, Gaber AO (2003) Diminished lung injury with vascular adhesion molecule-1 blockade in choline-deficient ethionine diet-induced pancreatitis. Surgery 133:186-196
26. Privratsky JR, Tilkens SB, Newman DK, Newman PJ (2012) PECAM-1 dampens cytokine levels during LPS-induced endotoxemia by regulating leukocyte trafficking. Life Sci 90:177-184

Publisher's Note Springer Nature remains neutral with regard to jurisdictional claims in published maps and institutional affiliations. 